\title{
REM Density in the Differential Diagnosis of Psychiatric From Medical-Neurologic Disorders: A Replication
}

\author{
Doug King, Hagop S. Akiskal, Helio Lemmi, William Wilson, Joe Belluomini, \\ and Boghos I. Yerevanian
}

Received May 5, 1981; revised version received August 21, 1981; accepted September 5, 1981.

\begin{abstract}
The discriminatory power of rapid eye movement (REM) density in 61 outpatients with medical, neurologic, and psychiatric disorders and 8 noncase controls was assessed. REM density was significantly lower in a group of patients with medical-neurologic disease as compared with psychiatric and control subjects without evidence for such disease. Furthermore, low scores discriminated depressions occurring in the context of somatic disease when compared with those in the absence of such disease. The differences between groups were not accounted for by age or sex. The cutoff REM density score of 12.56 , based on the $99 \%$ lower confidence limit of the noncase controls, provided the highest sensitivity $(0.82)$ without loss of specificity $(0.80)$. It was concluded that REM density may have merit as a general measure of diffuse central nervous system pathology, whether primary or secondary to widespread systemic disease. The findings of Kupfer's group are upheld and extended to a broader medical and neuropsychiatric population than in the original Pittsburgh study.
\end{abstract}

Key Words. REM density, sleep electroencephalogram, biological marker, neuropsychiatric disorders.

Over the past several years new strategies have been developed for exploring the usefulness of biological tests for making psychiatric diagnoses (Akiskal and Webb, 1978). Objective markers that appear ripe for clinical application include the dexamethasone suppression test and various electroencephalographic (EEG) sleep parameters (Kupfer, 1976; Akiskal, 1980; Carroll et al., 1981).

The search for EEG sleep correlates of psychiatric diagnostic categories has met with considerable success in the area of affective disorders (Kupfer and Foster, 1972; Kupfer et al., 1973; Foster et al., 1976; Kupfer, 1976; Kupfer et al., 1976, 1978; Gillin et al., 1979; Kupfer et al., 1980a, 1980b). In a series of publications the Pittsburgh group has shown that rapid eye movement (REM) latency and REM density distinguish primary from secondary depression. Thus, primary depressives have shorter REM

Doug King., M.D., is Assistant Professor of Psychiatry, University of Michigan, Ann Arbor, MI, USA. Hagop S. Akiskal, M.D., is Research Psychiatrist; Helio Lemmi, M.D., is Neurologist-in-Chief and Director; and Joe Belluomini is Technical Director, Sleep Disorders Center, Laboratory of Neurophysiology, Baptist Memorial Hospital, Memphis, TN, USA. Dr. Akiskal is also Professor of Psychiatry, Associate Professor of Pharmacology, and Director of Mood Clinic and Affective Disorders Program, and Dr. Lemmi is Clinical Professor of Neurology, University of Tennessee College of Medicine, Memphis. William Wilson, Ph.D., is Assistant Professor of Psychiatry, Vanderbilt University, Nashville, TN, USA. Boghos I. Yerevanian, M.D., is Assistant Professor of Psychiatry, University of Rochester, Rochester, NY, USA. At the time of this study, Drs. King and Yerevanian were Residents in Psychiatry at the University of Tennessee. (Reprint requests to Dr. H.S. Akiskal, Coleman Bldg., Rm. 3B09, 956 Court Ave., Memphis, TN 38163 USA.) 
latency and higher REM density, while secondary depressives show less severe disruption of sleep continuity and have a lower REM percentage. Furthermore, secondary depressives without medical disease have less sleep continuity disruption, higher REM percentage, and higher REM density than secondary depressives with medical disease (Kupfer and Heninger, 1972; Foster et al., 1976). Psychotic depressives tend to have shorter REM latency, more sleep continuity disturbance, and lower delta and REM percentages than their nonpsychotic counterparts (Kupfer et al., 1980a). The sleep of schizoaffectives more closely resembles that of psychotic depressives than of schizophrenics (Kupfer, 1976).

In general, REM latency has received considerably more attention as a potential biological marker of primary, melancholic, and psychotic depression than REM density (Kupfer et al., 1978). In the present study, we examined REM density in medically, neurologically, and psychiatrically ill patients and in noncase controls. Following the lead of Kupfer's group, we undertook to determine the usefulness of this measure for distinguishing these groups in a broader clinical population than in the original Pittsburgh study (Foster et al., 1976).

\section{Methods}

Subjects and Procedure. Sixty-one patients with at least 2 consecutive nights of sleep polysomnography were selected from clinical referrals to the Sleep Disorder Center. These patients all received the clinically standardized routine evaluation of our Center which includes: (1) being free of psychoactive drugs (including steroids) for at least 2-3 weeks before polysomnography; (2) the Stanford Sleep Questionnaire and comprehensive sleep history; (3) neurologic and medical history and examination; (4) auditory evoked potentials; (5) a routine EEG to rule out seizure disorder; (6) formal psychiatric evaluation; (7) Beck Depression Inventory and Minnesota Multiphasic Personality Inventory; and (8) sleep polygraphic study consisting of continuous EEG, submental electromyogram, and two channels of electro-oculogram (EOG), scored according to the method of Rechtschaffen and Kales (1968). When necessary, further clinical or laboratory information was obtained from referring physicians' records. Clinical information was abstracted "blind" to neurophysiologic status.

Diagnostic Criteria. Psychiatric diagnoses (Table $1 a$ ), following the Washington University schema (Feighner et al., 1972), were based on data obtained in semistructured interviews. Primary affective disorder $(n=18)$ refers to depression which occurs in the absence of nonaffective psychiatric diagnoses; four had bipolar and the remainder unipolar depression. Sccondary affective disorder $(n=9)$ refers to depressions associated with life-threatening or incapacitating somatic illnesses or superimposed on "nonorganic" psychiatric conditions that are considered validated by followup studies; these include anxiety (panic in $D S M-I I I$ ), phobic and obsessive-compulsive disorders, Briquet's disorder, sociopathy, schizophrenia, anorexia nervosa, transsexualism, and (ego dystonic) homosexuality. One patient who satisifed the criteria for primary depression except for mood change was considered undiagnosed (masked) depression. The group of psychiatric disorders (other than primary affective disorders) included: 3 with anxiety disorder (panic disorder in $D S M$ - III); 3 with Briquet's disorder; I with agoraphobia; 1 with obsessive-compulsive disorder; and 17 character-disordered patients who failed to meet Washington University criteria for specified validated diagnoses, but met DSMIII (American Psychiatric $\Lambda$ ssociation, 1980) criteria for one or more personality disorders. One patient categorized as an undiagnosed neurotic disorder had developed anxietyhypochondriacal symptoms following a central nervous system (CNS) febrile illness of unknown etiology.

Table $1 b$ lists the medical and neurologic conditions that were documented either by physical examination and/or laboratory findings or history. Ten patients had both psychiatric and medical-neurologic diagnoses. 


\section{Table 1. Frequencies of psychiatric diagnoses and medical-neurologic conditions}

\begin{tabular}{lc}
\hline a. Psychiatric diagnoses & $n$ \\
\hline Primary affective disorder & 18 \\
Secondary affective disorder & 9 \\
Undiagnosed (masked) depression & 1 \\
Anxiety (panic) disorder & 3 \\
Briquet's disorder & 3 \\
Agoraphobia & 1 \\
Obsessive-compulsive disorder & 1 \\
Undiagnosed (character) disorder & 17 \\
b. Neurologic and medical conditions &
\end{tabular}

\begin{tabular}{ll}
\hline Abnormal evoked potential study1 & 3 \\
Coronary artery disease & 3 \\
Localized frontal brain atrophy & 2 \\
Pellizius Merezbacher & 2 \\
Hypertensive cardiovascular disease & 2 \\
Hypertension & 1 \\
Pituitary adenoma (operated) & 1 \\
Encephalitis (history) & 1 \\
Meningitis (history) & 1 \\
Normal pressure hydrocephalus & 1 \\
Multiple sclerosis & 1 \\
Cerebrovascular accident (history) & 1 \\
Undiagnosed CNS febrile illness (history) & 1 \\
Ischemic labyrinthitis & 1 \\
Guillain-Barré (history) & 1 \\
Polymyalgia rheumatica & 1 \\
Diabetes mellitus & 1 \\
Abnormal glucose tolerance test & 1 \\
Chronic anemia & 1 \\
Gastric carcinoma & 1 \\
Emphysema & 1 \\
Chronic diverticulitis & 1 \\
\hline
\end{tabular}

1. Based primarily on prolonged (i.e., beyond 2 SD of laboratory norms) interpeak latencies of waves I, II, and V.

Two patients with chronic (5-10 years) "polydrug abuse" (including sedative-hypnotic drugs and alcohol) were not included in the overall analyses but considered separately in the discussion, because there was uncertainty as to whether they should be included in the psychiatric or medical-neurologic group.

Eight noncase controls, without evidence of psychiatric and medical-neurologic illness, provided a comparison group.

Hierarchical Categorization of Study Groups. For purposes of data analysis each patient was assigned to only one of the study groups (Table 2): group I, CNS disorders (including 
abnormal evoked potential study); group II, medical illnesses excluding specific CNS disorders; group III, primary affective disorder with no evidence of medical-neurologic disorder; group IV, all other psychiatric disorders; and group V, noncase controls. Since selection criteria did not exclude patients with more than one condition, the following rule was adopted for assigning patients with multiple diagnoses to groups: patients were assigned to group I if there was evidence of CNS disease or an abnormal evoked potential study regardless of whether they had other medical or psychiatric illnesses; those with medical illness but without CNS conditions were assigned to group II regardless of the presence or absence of psychiatric diagnoses; patients assigned to group III had primary affective disorder and no diagnosed somatic or other psychiatric illness; patients assigned to group IV had psychiatric illnesses other than primary affective disorder. Thus groups I, II, and IV included several patients with more than one diagnosis, whereas group III included patients with only one diagnosis. Group V comprised eight noncase controls. By this method the 59 patients and eight controls were sorted into five groups with unequal $n$ 's.

\begin{tabular}{|c|c|}
\hline Groups and diagnostic combinations & $n$ \\
\hline \multicolumn{2}{|l|}{ Group I $(n=14)$} \\
\hline CNS condition only & 7 \\
\hline CNS and medical condition & 2 \\
\hline $\begin{array}{l}\text { CNS and medical condition and primary } \\
\text { affective disorder }\end{array}$ & 1 \\
\hline $\begin{array}{l}\text { CNS condition and primary affective } \\
\text { disorder }\end{array}$ & 1 \\
\hline $\begin{array}{l}\text { CNS condition and other psychiatric } \\
\text { disorder }\end{array}$ & 3 \\
\hline \multicolumn{2}{|l|}{ Group II $(n=8)$} \\
\hline Medical condition only & 3 \\
\hline $\begin{array}{l}\text { Medical condition and other psychiatric } \\
\text { disorder }\end{array}$ & 5 \\
\hline \multicolumn{2}{|l|}{ Group III $(n=18)$} \\
\hline Primary affective disorder only & 16 \\
\hline \multicolumn{2}{|l|}{ Group IV $(n=21)$} \\
\hline Other psychiatric disorder only & 16 \\
\hline $\begin{array}{l}\text { Other psychiatric disorders and secondary } \\
\text { depression }\end{array}$ & 5 \\
\hline \multicolumn{2}{|l|}{ Group V $(n=8)$} \\
\hline Noncase controls & 8 \\
\hline
\end{tabular}

REM Density Measurement. REM density data represent the mean of nonadaptation nights. REM periods are scored in 1-minute epochs. The actual rapid eye movements are scored when they show amplitudes greater than $25 \mu \mathrm{V}$. REM density represents the actual number of minutes of rapid eye movements that occur during a REM period. REM density values are calculated by the following formula: 
$\frac{\text { total minutes of rapid eye movements }}{\text { total minutes of REM sleep }} \times 100$

Statistical Analysis. An unweighted means analysis of variance for unequal $n$ 's was performed on REM density measures for the five groups. Scheffé's test of multiple comparisons was performed to assess differences of REM density means between group pairs and to assess the difference between combined groups I and II and combined groups III, IV, and V. An analysis of variance and Scheffé comparisons were used to test for age differences between groups, and a chi square was used to test for sex differences between groups (Winer, 1971). Pearson correlations were determined for age and REM density and for sex and REM density.

The sensitivity and specificity of REM density for discriminating subjects with medicalneurologic illness (groups I and II) from subjects without medical-neurologic illness (groups III, IV, and V) were determined by deriving cutoff points from noncase control data. Three cutoffs were obtained by calculating the $95 \%, 99 \%$, and $99.9 \%$ one-tailed confidence intervals (standard error of the mean times the respective $Z$ scores for each confidence interval). The total sample of REM density scores was thus dichotomized (once for each confidence interval) into: (a) scores falling at or above the cutoff and (b) scores falling below the cutoff. Chi squares were performed for each data set generated by the three cutoffs.

\section{Results}

For the five groups under study chi square did not show significant sex-ratio differences (Table 3). On the other hand, analysis of variance revealed that groups differed significantly with respect to age $(p<0.05)$. Scheffé comparisons indicated that the control group was significantly younger than groups I and II; furthermore group II was significantly older than group IV.

Table 3. Age and sex distribution for subject groups

\begin{tabular}{lrccc}
\hline & & & \multicolumn{2}{c}{ Age $^{2}$} \\
\cline { 5 - 5 } Groups & $n$ & M:F ratio & Mean & SD \\
\hline I & 14 & $4: 3$ & 47.0 & 20.23 \\
II & 8 & $4: 1$ & 57.5 & 11.29 \\
III & 16 & $5: 11$ & 44.5 & 17.35 \\
IV & 21 & $8: 13$ & 39.4 & 13.61 \\
V & 8 & $5: 3$ & 29.3 & 8.68 \\
\hline
\end{tabular}

1. $\chi^{2}=6.102, d f=4, p=N S$.

2. ANOVA, $F=3.381$, $d f=4,62, p<0.05$.

Significant Scheffé comparisons (age:

I vs. $V, \rho<0.05$

II vs. IV, $p<0.05$

II vs. $V, p<0.05$

Pearson correlations between age and REM density $(r=0.016)$ and sex and REM density $(r=0.164)$ did not indicate a statistically significant association.

Table 4 summarizes the results of analysis of variance for REM density for all groups. The overall analysis was significant $(p<0.01)$. Scheffé comparisons indicated that group II differed significantly $(p<0.05)$ from groups III and IV and that the 
combined groups (I and II) differed significantly from the combined groups (III, IV, and V) $(p<0.05)$. Further analyses (Table 5) revealed that depressed patients with medical-neurologic diagnoses had significantly lower REM density than depressed patients without medical-neurologic diagnoses $(p<0.01)$.

Table 4. REM density comparisons between groups

\begin{tabular}{lllllc}
\hline & \multicolumn{5}{c}{ Groups } \\
\cline { 2 - 6 } & \multicolumn{1}{c}{ I } & II & III & IV & V \\
\hline$n$ & 14 & 8 & 16 & 21 & 8 \\
Mean & 12.33 & 4.51 & 20.23 & 20.95 & 18.03 \\
SD & 11.96 & 3.42 & 10.33 & 12.43 & 6.65 \\
\hline
\end{tabular}

ANOVA, $F=4.578, d f=4,62, p<0.01$

Significant Scheffé comparisons:

II vs. III, $p<0.05$.

II vs. IV $p<0.05$.

$(I+I I)$ vs. (III + IV + V), p<0.05.

Table 5. REM density comparison between depressed patients with neurologic or medical illness (D+) and those without evidence of neurologic or medical illness (D-)

\begin{tabular}{lll}
\hline & (D+) & (D-) \\
\hline$n$ & 6 & 21 \\
Mean & 3.57 & 21.87 \\
SD & 2.48 & 13.02 \\
\hline
\end{tabular}

ANOVA, $F=10.53, d f=2,26, p<0.01$.

To assess the diagnostic usefulness of REM density scores for discriminating medical-neurologic patients from those without such illnesses, a scries of REM density "cutoff" points were derived based on the mean of the normal control group $\left(\overline{\mathrm{X}}_{\mathrm{RD}}=\right.$ 18.03). Table 6 is based on the one-tailed confidence intervals $(95 \%, 99 \%, 99.9 \%)$ obtained by multiplying the standard error of the mean (2.35) of the noncase controls by the $Z$ scores for each of the intervals yielding the following cutoff values: 14.16; $12.56 ; 10.77$. Chi-square tables were developed for each of these values, and diagnostic sensitivities and specificities for each cutoff point were computed. Sensitivities ranged from 0.73 to 0.82 , and specificities from 0.71 to 0.89 . These figures indicated that regardless of the cutoff used, the majority of somatically ill subjects had "low" (i.e., below the cutoff) REM density, while most subjects without such illness had "normal" or "high" REM density (i.e., values above the cutoff). 


\begin{tabular}{|c|c|c|c|c|c|c|}
\hline Cutoff & 14.16 & $(95 \%)$ & 12.56 & $(99 \%)$ & 10.77 & $(99.9 \%)$ \\
\hline$\overline{x^{2}}$ & 16.65 & & 23.47 & & 26.07 & \\
\hline Sensitivity & 0.82 & & 0.82 & & 0.73 & \\
\hline Specificity & 0.71 & & 0.80 & & 0.89 & \\
\hline
\end{tabular}

1. $d f=1, p<0.01$.

\section{Discussion}

The results of the present study revealed a significant inverse relationship between phasic REM activity (REM density) and the presence of neurologic and/or medical illness. Thus a rank ordering of the mean REM densities for the groups yielded: II $<\mathrm{I}$ $<$ V $<$ III $<$ IV $(4.51<12.33<18.03<20.23<20.95)$. The mcdical group significantly differed from the primary affective and other psychiatric groups. The difference between the medical and control groups approached but did not reach significance. I he neurologic group did not differ significantly from any other group. However, when medical and neurologic groups were combined, they were significantly different from the combined psychiatric and control groups. Since the groups differed in age, it was necessary to examine the impact of age on REM density. Pearson correlation ( $r=$ 0.016) for age and REM density suggested that in our sample very little of the REM density variance could be accounted for by age. Further, a Pearson correlation coefficient of 0.164 for REM density and sex indicated that REM density and sex were not strongly associated.

The overall trend of the data was, therefore, for subjects with medical-neurologic illness to have lower REM density values than subjects without such illness (primary affectives, other psychiatric, and normal controls). This bimodal tendency was further supported when the REM density scores of patients with clinical depression were analyzed: depressed subjects with a medical-neurologic illness had low REM density $\left(\overline{\mathrm{X}}_{\mathrm{RD}}=3.57\right)$ compared to depressed subjects with no associated inedical or neurologic illness $\left(\mathrm{X}_{\mathrm{RD}}=21.87\right)$.

The failure to find a statistically significant difference between the medical group and controls, and between the CNS group and psychiatric and control groups, requires further comment. An inspection of the individual scores of the medical group revealed that only one subject had a REM density value (11\%) which fell within the $99.9 \%$ one-tailed confidence intervals of the noncase control distribution. Thus, there was little overlap between the distribution of scores for the two groups, but because of large total variance of the sample, statistical analysis (Scheffé comparison) did not reach significance.' ${ }^{1}$ Further, inspection of the individual scores for the CNS group reveals four scores which fell at or well above the upper $0.1 \%$ of the noncase control

1. A $t$ test, a less conservative test than the Scheffé comparison, was calculated comparing group II and group $\mathrm{V}$. The difference was significant at the $p<0.005$ level of confidence. 
distribution ( $99.9 \%$ confidence interval, one-tailed-upper portion of the distribution = 25.29). Statistically these scores were as "aberrant" as the "low" REM density scores. Whether "high" REM density is clinically significant is yet to be determined. Nevertheless, it is interesting that in the CNS group there appear to be two distinct clusters of scores: (1) "low": $n=10, \overline{\mathrm{X}}_{\mathrm{RD}}=5.6, \mathrm{SD}=3.47$; and (2) "high": $n=4 ; \overline{\mathrm{X}}_{\mathrm{RD}}=29.25, \mathrm{SD}=$ 7.14. The diagnoses of subjects with these high REM densities were: local frontal atrophy, REM density $=30$; local frontal atrophy, REM density $=25$; meningitis by history, $\operatorname{REM}$ density $=23$; and multiple sclcrosis, REM density $=39$. Thus, the CNS group appears to be heterogeneous, and these few aberrantly high scores (in an otherwise low REM density CNS group) may explain why the CNS group was not significantly different from the psychiatric and control groups. Studies with larger sample size and a wide variety of CNS lesions are needed to determine whether this bimodality of scores for the CNS group is valid or artifactual; and to determine what type of CNS lesion is associated with a significant reduction in REM density. There is some evidence to suggest that the frontal cortex has an inhibitory effect on rapid eye movements, and the visual cortex is facilitatory (Appenzeller and Fischer, 1968). Accordingly, destructive lesions of the frontal cortex are expected to result in increased rapid eye movement. This hypothesis is consistent with the finding of increased REM density in our two patients with localized frontal atrophy. Our sample also included two patients with cerebellar disease. Sleep polygraphic studies of similar patients with olivopontocerebellar degeneration have shown decreased REM density and REM percent (Neil et al., 1980). Because cerebellar lesions by themselves do not affect sleep mechanisms, the changes in REM density in patients with cerebellar disease might reflect dysfunction in caudal pontine (including locus ceruleus) nuclei.

In our series, several subjects with diagnosed systemic but without manifest (diagnosable) CNS disease showed low REM density $(<10.77)$. These included three patients with coronary artery disease; one patient with hypertensive cardiovascular disease and an abnormal glucose tolerance test; one patient with chronic anemia; one patient with gastric carcinoma. None of these subjects had history or evidence of ccrebrovascular abnormality, yet all had extremely low REM densitics. The finding of low REM density in these patients may extend the range of pathology which is reflected in diminished phasic REM activity. In studies by the Pittsburgh group (Foster et al., 1976; Kupfer et al., 1980a), patients with diagnoses of cardiovascular disease were not specifically mentioned.

The usefulness of REM density in differentiating patients with medical or neurologic illnesses from psychiatric patients without concomitart medical-neurologic illnesses and from normal controls was supported by chi-square tests using different cutoff points for "low" REM density derived from the noncase control subjects' scores. For each of the cutoff values, low REM density was strongly correlated with the presence of medical-neurologic illness. As expected, the lowest cutoff point (10.77) yielded the highest sensitivity and the lowest specificity, while the reverse was true for the highest cutoff point (14.16). The 12.56 cut off yielded high specificity $(0.80)$ without loss of sensitivity (0.82).

Our original sample included two patients (excluded from analyses) with a primary diagnosis of chronic ( 5 to 10 years) polydrug abuse, including sedative-hypnotics and ethanol; they had been free of these drugs for a 1-month period before the sleep study. 
These patients had low REM density values (4\% and $2.7 \%$ ). In the analyzed sample, patients with REM density in this low range invariably had a medical or CNS illness. This finding is consistent with other evidence linking chronic sedative-hypnotic and alcohol abuse to CNS dysfuncton (Ballenger and Post, 1978; Judd and Grant, 1978).

The data from our outpatient sample support and extend the findings generated by Kupfer's laboratory with regard to low REM density in secondary depressions in medically ill inpatients. It appears that low REM density is associated with a wide variety of somatic conditions regardless of the presence or absence of affective disorder. It would be premature to speculate about the precise neurophysiologic mechanisms responsible for low REM density. However, the available data suggest that disturbances which "diffusely" affect brain tissue may be more likely to result in low REM density than lesions with localized impact; caudal pontine lesions might be the exception to this generalization. It is possible that widespread systemic diseases may diffusely affect brain function in subtle ways (e.g., decreased REM density) which are not otherwise manifest in "gross" neurologic testing. Since the visual system is widely distributed in the brain, it is to be expected that a diverse and heterogeneous group of both CNS and systemic diseases (as well as pontine lesions with direct impact on eye movements) would affect phasic REM activity. Hence, low REM density may serve as a general indicator of diffuse CNS pathology of diverse origin whether endogenous, or exogenously produced.

Work on a large sample is in progress in our laboratory to explore further the common somatic denominator of conditions associated with low REM density and the usefulness of this measure in the differential diagnosis of neuropsychiatric, medical-psychiatric, and "functional" psychiatric disorders.

Acknowledgment. Sharon Burt, Edward Kish, Lisa Milligan, and Phil Vance provided technical assistance.

\section{References}

Akiskal, H.S. External validating criteria for psychiatric diagnosis: Their application in affective disorders. Journal of Clinical Psychiatry, 41, 6 (1980).

Akiskal, H.S., and Webb, W.L., eds. Psychiatric Diagnosis: Exploration of Biological Predictors. Spectrum Publications, New York (1978).

American Psychiatric Association. DSM-III: Diagnostic and Statistical Manual of Mental Disorders. 3rd ed. APA, Washington, D.C. (1980).

Appenzeller, O., and Fischer, A.P. Disturbances of rapid eye movements during sleep in patients with lesions of the nervous system. Electroencephalography and Clinical Neurophysiology, 25, 29 (1968).

Ballenger, J.C., and Post, R.M. Kindling as a model for the alcohol withdrawal syndromes. British Journal of Psychiatry, 133, I (1978).

Carroll, B.J., Feinberg, M., Greden, J.F., Tarika, J., Albala, A.A., Haskett, R.F., James, N.M., Kronfol, Z., Lohr, N., Steiner, M., de Vigne, J.P., and Young, E. A specific laboratory test for the diagnosis of melancholia: Standardization, validation and clinical utility. Archives of General Psychiatry, 38, 15 (1981).

Feighner, J.P., Robins, E., Guze, S.B., Woodruff, R.A., Winokur, G., and Munoz, R. Diagnostic criteria for use in psychiatric research. Archives of General Psychiatry, 26, 57 (1972).

Foster, F.G., Kupfer, D.J., Coble, P., and McPartland, R.J. Rapid eye movement sleep density: An objective indicator in severe medical-depressive syndromes. Archives of General Psychiatry. 33, 1119 (1976). 
Gillin, J.C., Duncan, W., Pettigrew, K.D., Frankel, B.L., and Snyder, F. Successful separation of depressed, normal, and insomniac subjects by EEG sleep data. Archives of General Psychiatry, 36, 85 (1979).

Judd, L.L., and Grant, I. Intermediate duration organic mental disorder among polydrug abusing patients. Psychiatric Clinics of North America, 1, 153 (1978).

Kupfer, D.J. REM latency: A psychobiological marker for primary depressive disease. Biological Psychiatry, 11, 159 (1976).

Kupfer, D.J., Broudy, D., Coble, P.A., and Spiker, D.G. EEG sleep and affective psychosis. Journal of Affective Disorders, 2, 17 (1980a).

Kupfer, D.J., and Foster, F.G. Interval between onset of sleep and rapid-eye-movement sleep as an indicator of depression. Lancet, September 30, 684 (1972).

Kupfer, D.J., Foster, F.G., Coble, P., McPartland, R.J., and Ulrich, R. The application of EEG sleep for the differential diagnosis of affective disorders. American Journal of Psychiatry, 135, 69 (1978).

Kupfer, D.J., Foster, F.G., and Detre, T.P. Sleep continuity changes in depression. Diseases of the Nervous System, 34, 192 (1973).

Kupfer, D.J., Foster, F.G., Reich, L., Thompson, K.S., and Weiss, B. EEG sleep changes as predictors in depression. American Journal of Psychiatry, 133, 622 (1976).

Kupfer, D.J., and Heninger, G.R. REM activity as a correlate of mood changes throughout the night: Electroencephalographic sleep patterns in a patient with a 48 -hour cyclic mood disorder. Archives of General Psychiatry, 27, 368 (1972).

Kupfer, D.J., Spiker, D.G., Coble, P.A., Neil, J.F., Ulrich, R., and Shaw, D.H. Depression, EEG sleep, and clinical response. Comprehensive Psychiatry, 21, 212 (1980b).

Neil, J.F., Holzer, B.C., Spiker, D.G., Coble, P.A., and Kupfer, D.J. EEG sleep alterations in olivopontocerebellar degeneration. Neurology, 30, 660 (1980).

Rechtschaffen, A., and Kales, A., eds. A Manual of Standardized Terminology, Techniques and Scoring System for Sleep Stages of Human Subjects. National Institutes of Health Publication No. 204, Superintendent of Documents, U.S. Government Printing Office, Washington, DC (1968).

Winer, B.J. Statistical Principles in Experimental Design. 2nd ed. McGraw Hill, New York (1971). 